mind similar to such a process as this. It can be made conceivable only by a mechanical analogy, if at all.

If a ball is freely suspended from the roof of a railway carriage, it will receive a shock sufficient to move it, when the carriage is set in motion: and the magnitude and direction of the shock thus given to the ball will depend on the magnitude and direction of the force with which the carriage begins to move. While the carriage is in uniform motion the ball will be relatively at rest; and every change in the velocity of the motion of the carriage, and of its direction, will give a shock of corresponding magnitude and direction to the ball. Now, it is conceivably quite possible, though such delicacy of mechanism is not to be hoped for, that a machine should be constructed, in connection with a chronometer, for registering the magnitude and direction of all these shocks, with the time at which each occurred; and from these data-the direction of the shock indicating the direction of the motion of the carriage, the magnitude of the shock indicating its velocity, and the interval of time between two shocks indicating the time during which the carriage has run without change of velocity or direction-from these data the position of the carriage, expressed in terms of distance and direction from the place from which it had set out, might be calculated at any moment. The automatic register of the journey may be conceived as exactly resembling the records of the velocity and direction of the wind produced by one of Robinson's or Beck's self-registering anemometers, where one pencil-mark indicates the direction of the wind, at any past hour, and another its velocity.

Further, it is possible to conceive the apparatus as so integrating its results as to enable the distance and direction of the point where the journey began to the point it has reached, that they can be read off, without any calculation being needed:- - a hand on a dial pointing to the direction expressed in degrees of the circle, and the distance being shown in figures expressing miles and decimals of a mile.

Now, I suppose such an integrating process as this (though of course not by any similar mechanism) to be effected in the brain of an animal unconsciously, and that the animal has the power of reading off the result--that is to say, bringing it into consciousness when wanted.

Old Forge, Dunmurry, Co. Antrim, April I I

Joseph John Murphy

\title{
The Role of Lighting in Aids to Marine Navigation
}

\section{P. Clissold}

L. G. Reynold's interesting article (Journal $30, x 08$ ) gives rise to a question. Why, on the new charts is the light range given as the luminous or nominal range, instead of, as formerly, the geographical range (for a height of eye of i $5 \mathrm{ft}$ ) ? This, from the point of view of the practical navigator, seems a retrograde step for we can no longer see from a glance at the chart the approximate 
range at which we may expect to sight a light. The luminous range is almost always greater than the geographical range and reference must be made to the Geographical Range Table in the Admiralty List of Lights to find out what the latter will be.

\section{The Hydrographic Department comments:}

Commander Clissold contends that the changing of light range on the chart from the geographical range to luminous or nominal range is a retrograde step for the practical navigator. Whilst sympathizing with Commander Clissold's view the Hydrographer of the Navy cannot agree.

The decision to adopt nominal or luminous range both on the chart and in the light lists was taken after protracted international debate in which the needs of practical navigation were comprehensively considered. The Hydrographer contended that to quote the range of a light on the mere basis of an observer's height of eye of $15 \mathrm{ft}$ was unrealistic since this height was no longer applicable to the majority of ocean-going ships. Thus any observer whose height of eye was not this magic i $5 \mathrm{ft}$ was already having to look in the tables in the front of the light list (or other appropriate tables) to find the range at which the light would be raised.

The long established practice of charting the lesser of geographical and luminous ranges made comparison between charted lights well nigh impossible. It was felt that a measure of the practical strength of the light was needed and thus the use of one type of range-nominal range-gives more realistic basis for comparison and represents a mean performance figure for global use; as $\mathrm{Mr}$. Reynolds points out on page i i 5 of the January Journal.

The sighting range of a light depends on so many variable factors, of which the observer's height of eye is but one, that it could be argued that to show any range at all on the chart is to present the mariner with misleading information. However, nominal range, or luminous range for those countries which use a greater transmission factor than 0.74 , does provide a much more consistent figure upon which to base the calculation of the sighting range of a light. There is no short cut to the determination of this range; nor has there been since the navigation platform moved upwards from a representative i $5 \mathrm{ft}$ height of eye. When the changeover to nominal range was made the attention of mariners was drawn to the preliminary pages in the Admiralty List of Lights which form an essential adjunct to the chart for obtaining sighting ranges.

\section{A Polar Compass}

\section{Captain F. G. Wolff}

THE basic premise of compass adjusting (that is compensatiun aboard ship for magnetic fields caused by the vessel itself and the determination of residual deviation) is that a reference direction must be available during the operation. Normally visual ranges or the azimuth of a celestial body-usually the Sun-are used, but in some areas and climates reduced visibility often does not permit the 